\title{
Identifying Predictors of Patient Safety Competency Based on Teamwork and Psychological Safety among emergency nurses.
}

\section{Aghil Habibi Soola}

Ardabil University of Medical Sciences

Mehdi Ajri-Khameslou

Ardabil University of Medical Sciences

Alireza Mirzaei ( $\square$ alirezamirzaei9015@gmail.com )

Ardabil University of Medical Sciences

Zahra Bahari

Ardabil University of Medical Sciences

\section{Research Article}

Keywords: Patient safety competency, Teamwork, Team structure, Leadership, Psychological Safety Emergency nurses

Posted Date: November 8th, 2021

DOI: https://doi.org/10.21203/rs.3.rs-1050803/v1

License: (c) (i) This work is licensed under a Creative Commons Attribution 4.0 International License. Read Full License 


\section{Abstract}

Aims

To identify factors that predict the patient safety competency of emergency nurses.

\section{Background}

The role of emergency nurses is to provide high-quality health care to patients and ensure their safety. The patient safety competency includes the absence of unnecessary or potential harm when providing health care to patients. In providing health care, effective teamwork can affect patient safety and outcome. Psychological safety is essential to effective teamwork. Psychological safety allows health care workers to accept the interpersonal risks needed to perform effective teamwork and maintain patient safety.

\section{Methods}

This descriptive correlation study included 254 emergency department nurses from five educational hospitals using convenience sampling. Patient Safety in Nursing Education Questionnaire was used to measure the patient safety competency, the teamwork questionnaire to examine the teamwork, and Edmondson psychological safety questionnaire was used to measure psychological safety. Descriptive statistics, $\mathrm{t}$-Test, one-way ANOVA, Pearson's correlation, and Stepwise multiple regression were performed using SPSS 14.0.

\section{Results}

The findings of the present study showed that the level of patient safety competency among emergency nurses is at a desirable level. Of the 18 independent variables evaluated in the multiple regression analysis, seven had a significant effect on the patient safety competency of emergency nurses $\left(R^{2}: 0.39\right.$, $p<.001)$.

\section{Conclusions}

The patient safety competency of emergency department nurses was primarily related to the structure and leadership of the team and secondary to psychological safety and experience in patient safety activity. The results demonstrated that policymakers and hospital managers should improve and enhance team structure and leadership via supervision and cooperation with the nursing staff. The development of training programs in the field of patient safety activities, improvement, and increase of psychological safety at the levels of the nursing units is essential to increase patient safety competencies in the emergency nursing program.

\section{Introduction}


The patient's safety is one of the most important health care systems which was considered in recent years (1). Though error and adverse events occur in hospital environments with significant frequency, but in units such as Emergency Department, where the patients have more bad situations, the prevalence is higher and varied from 4 to $68 \%$ (2). The patient's safety is a new concept in Iranian hospitals.

Nevertheless, since 2009 in the Iranian healthcare system, initiatives such as hospital accreditation, clinical sovereignty, and patient safety hospitals were presented as a framework for improving safety and quality of care in the hospital (3). Unpredictability and the widest nature of emergency departments, the critical condition of most patients, and large volumes of work due to the inadequacy of the patient and nurse ratios converts the emergency department to a high-risk area for patient adverse events. Teamwork in the emergency is a fundamental necessity compared to other parts of the hospital because deciding in the emergency is quick and accurate (4).

Teamwork is very important due to the critical work environment, a large number of staff, and the complexity of work in the emergency department (ED) (5). In the field of health, teamwork refers to a dynamic process that involves two or more health professionals who can communicate with each other, share common goals, and commit to achieving those goals. They also have complementary skills and demonstrate their concerted efforts to evaluate, care for and treat patients via collaboration, open communication, and joint decision-making under the supervision of team leader (6). Teamwork was suggested as a solution to improve the quality of care and safety of patients in the emergency department. Teamwork is defined as 'joint or coordinated efforts by a group of individuals acting as a team or in the common interest (7). Teamwork is essential for patient safety, and nurses play an important role to prevent adverse events by increasing the error report (8). Teamwork based on staff coherence reduces medical and nursing errors, resulting in more patient satisfaction and improved healthcare. Effective teamwork improves the patient's efficiency and safety, leads to a healthier and happier workplace, and reduces job burnout among healthcare professionals (9). According to the results of qualitative research, emergency nurses believe that group work may help increase the level of efficiency and safety of patient care in the emergency department (10). Considering the increase in demand, emergency departments need to improve efficiency while providing safe and effective care. Providing efficient and quality health care is affected by interactions among physical structure, processes, and emergency department results (11). Teamwork was proposed as an effective approach to care teams in the treatment process of the emergency department (4).

Despite all the evidence for the positive effects of teamwork on patient quality of care and safety, teamwork may not always lead to the desired results. Teamwork sometimes leads to negative conflicts among team members or among different teams, which ultimately leads to dysfunctional relationships among employees and disregard and disrespect for other team members. As a result, it leads to negligence in patient care. This is because nurses' attitudes toward teamwork as well as interaction and communication with each other and other members of the patient care team are different. (5). In health care teams which need effective teamwork to coordinate safe patient care in a very complex, dynamic, and high-risk environment, psychological safety is a very important factor (12). 
Psychological safety is defined as the conditions under which team members feel safe to take risks, discover new ideas, and challenge the status quo (13). Edmondson defines psychological safety as a common belief among team members who create a safe range for individual risk-taking. In fact, the team is ensured that group members are not blamed for exporting their thoughts. High psychological safety represents team atmosphere, interpersonal trust, and mutual respect. Psychological safety reduces the fear of rejection and supports active participation, creates an environment that team members probably recognize errors and resolve disadvantages and shortcomings $(13,14)$. Positive interpersonal relationships cause psychological safety. In health care teams, having positive relationships, effective patterns, and better teamwork conditions encourages health care professionals to talk about safety (12). Psychological safety of team members strengthens creativity and active participation in teamwork. Also, providing psychological safety to nurses increases patient safety and quality of care and it lack leads to increased psychological stress, absenteeism, endangering patient safety and reducing job satisfaction in Nurses $(13,14)$. Psychological safety significantly affects teamwork, team learning, organizational understanding, teamwork competency and patient safety competency (4).

Patient safety competency in nurses is crucial because nurses are responsible for the 24-hour care of patient. Patient safety competency refers to the attitudes, skills, and knowledge that health care workers must have to protect patients from unnecessary risks and hazards (4). According to Tella et al., the main content of the patient's safety in contemporary nursing education includes the competency to prevent patient safety accidents (attitude), the creation of patient safety competency (knowledge), and the competency to act after the error (skill) (15). A study showed that the weakness in the emergency department patient safety is due to the lack of optimal reports of incidents and lack of teamwork in the units, and the transfer of patients (16).

In the study by Han et al., reporting of patient safety adverse events, situation monitoring, and psychological safety were the main factors predicting patient safety competency (4). therefore, further studies are needed to evaluate the effects of a training program designed to increase patient safety competency in emergency nurses. Today, the emergency department is considered a multidisciplinary unit and, considering the need for cooperation among several people with different professionals in the patient's care process, as well as limited and contradictory studies concerning the patient safety competency and its effective factors in Iran; on the other hand, lack of a specific study in this context in the emergency unit; this study aimed to evaluate the relationship between Patient Safety Competency whit teamwork and psychological safety among Emergency Nurses

\section{Methods}

\section{Study design}

This descriptive correlation study was designed as a questionnaire-based, cross-sectional analysis.

\section{Study setting and participants}


The setting for this study was five emergency departments of Ardabil Teaching Hospitals, northwest of Iran. Among these five hospitals, two hospitals provide generalized services for their patients, and three hospitals provide specialized services. The total number of inpatient bed count in the emergency department in the studied hospitals was 220 beds. The Inpatient Bed Occupancy Rate before the health system reform plan was $75 \%$ on average. This rate reaches $95 \%$ and sometimes $100 \%$ after the start of the health system reform plan. In emergency departments, the division of labor is done in writing and as a case method. The study population included all nurses working in the emergency departments of generalized and specialized hospitals of Ardabil University of Medical Sciences. Ardabil city has five educational hospitals, including generalized centers and 2 specialized centers. The total number of nurses working in the emergency departments of the studied hospitals was 320 nurses. The inclusion criterion was having at least 12 months of experience in the emergency department, a bachelor's or higher degree in nursing, and a tendency to participate in the study. New graduate nurses with less than one year of experience were excluded from the study. Paper-based questionnaires were distributed by the researchers among 290 nurses eligible to participate in the study. Finally, 254 emergency nurses were enrolled in the study using convenience sampling.

\section{Study tools}

Data collection tools included the demographic and professional information form of participants and patient safety competency questionnaire, teamwork, and psychological safety.

\section{Demographic information form}

Questionnaire of demographic and professional information includes the items of age, gender, level of education, type of hospital, type of emergency department, number of night shifts per month, the total number of years of work experience, the total number of years of emergency work experience, job satisfaction, training on the patient safety (yes/no), experienced in patient safety activities and reporting of patient safety adverse events (adverse event reported by self and those reported by others). Job satisfaction was measured using a question (how satisfied are you about your job?) on the 5-degree Likert scale ( 1 = high dissatisfaction, 5 = high satisfaction) were measured.

\section{Patient safety competency}

One of the valid tools in measuring safety competency is the Patient Safety in Nursing Education Questionnaire (15). This scale evaluates the understanding of nurses from the patient's safety based on three categories: Academic conditions (19 items), patient safety in clinical conditions (17 items), and patient safety competency (14 items). To measure the patient safety competency, the third part of the safety questionnaire was used in nursing education. Langari et al. (17) examined the validity and reliability of the third part of the Patient Safety in Nursing Education Questionnaire. In the study of Langri et al., Exploratory analysis factor of three factors in the patient safety competency that forms the reliability of the patient; creating patient safety competency (knowledge); performance competency after the error (skill); and competency to prevent patient safety incidents (attitude). Its validity was reported at 
0.89 using Cronbach's alpha method (17). After the permission to use the questionnaire concessionaire (15), the tool was translated to the Persian language forward and backward by two English experts. To determine the content - the validity of the questionnaire from the content validity index (CVI) was used. For this purpose, the questionnaire was given to 12 nursing faculty members of Ardabil University of Medical Sciences and was examined in terms of relevance, simplicity, and clarity of statements, with a validity index of $91 \%$. Cronbach's alpha coefficient of 0.90 also showed the reliability of the questionnaire. This questionnaire consists of three subgroups of knowledge (4 items), skill (4 items), and attitude (6 items) and follows the Likert scale 4 (completely disagree $=1$ to completely agree $=4$ ). The validity of this questionnaire in this study was estimated at 0.90 by using Cronbach's alpha

\section{Teamwork}

A teamwork questionnaire (STEPPS (T-TAQ) was developed by Baker et al. (18) to identify people's attitudes about teamwork. The tool has 30 questions in five areas. The team structure (six items), leadership (six items), mutual support (six items), Situation monitoring (six items), and communication (six items). The items in the questionnaire are scores using the 5-option Likert scale of completely disagree (1) to completely agree (5). Four questions are scored in reverse, three questions in mutual support (questions 20,21, and 24) and one question in communication (question 30) (18). This questionnaire was translated in Iran by Najafi et al. (19), and Cronbach's alpha of 0.80 has been obtained. Cronbach's alpha was 0.93 in this study.

\section{Psychological safety Questionnaire}

The psychological safety scale was designed by Edmondson (20) to assess psychological safety in teamwork. This questionnaire consists of 7 items, seven-point Likert scale questions (completely disagree $=1$, completely agree $=7$ ). Questions 1,3 , and 5 are scored in reverse, and higher scores on the psychological safety Questionnaire indicate the respondent perceives a higher level of team psychological safety (20). This questionnaire was translated and psychometric in Persian by Shams in Iran, and its Cronbach's alpha was 0.81 obtained (21). The validity of this questionnaire in this study was 0.57 using Cronbach's alpha.

\section{Data collection and ethical considerations}

To do the work, first this study with the ethical code ...... was approved by the ethics committee of ...... University of Medical Sciences. The researchers then referred to Imam Khomeini, Imam Reza, Fatemi, Bouali, and Alavi hospitals in Ardabil and were introduced to emergency nurses by the nursing offices of the mentioned centers. After explaining the research objectives and emphasizing the completeness and completeness of the questionnaires and confidentiality Availability of information, first informed written consent was obtained from nurses eligible to participate in the study; then, the paper version of the questionnaire was distributed by the researchers to the emergency nurses who had given their written consent to attend the study. Participants were also informed of the right to refuse 
participation or the possibility of canceling at any stage of the research without any penalty. Information was collected from February 1 to 1 April 2021.

\section{Data analysis}

Data were analyzed using SPSSV14 software. The level of teamwork, psychological safety, and patient safety competency in emergency nurses were analyzed using descriptive statistics. The interactions of teamwork, psychological safety, and patient safety competency of emergency nurses were analyzed using Pearson correlation. The relationship between patient safety competency and demographic characteristics was investigated using an independent t-Test, ANOVA, and Pearson correlation. The predictive factors of patient safety competency from emergency nurses using multiple stepwise regression were investigated.

\section{Results}

290 nurses completed 254 surveys. The response rate was $87 \%$ (254/290). The demographic characteristics of respondents were shown in Table 1.

\section{Descriptive statistics and relationship between patient safety competency and teamwork and psychological safety}

Table 2 shows the descriptive statistics and the relationship among patient safety competency, teamwork, and its subgroups, and psychological safety of emergency nurses. The mean and standard deviation of patient safety competency, teamwork, and psychological safety was $2.97 \pm 0.54$ with a score range from (1-4), $3.60 \pm 0.56$ with a score range from (1-5), $27.04 \pm 4.67$ with a score range from (9-39, respectively. There was a positive correlation between the mean score of patient safety competency with teamwork $(r=0.4873, P<.001)$ and psychological safety $(r=0.167, P<.001)$. Furthermore, the patient safety competency had a positive correlation with leadership, communication, mutual support, situation monitoring, and team structure $(P<.001)$.

\section{Relationship between patient safety competency and general characteristics of emergency nurses}

Table 3 shows the relationship between patient safety competency and general characteristics of emergency nurses. Patient safety competency was significantly negatively correlation to the type of hospital $(t=-5.224, p<.001)$. Patient safety competency with age $(r=0.170, p=0.007)$, job satisfaction ( $F$ $=3.475, p=0.009)$, education level $(t=3.290, p=0.001)$, type of employment $(F=2.678, p=0.048)$, type of emergency $(t=3.602, p=0.029)$, Training in patient safety $(t=3.256, p=0.001)$, Experience in patient safety activity $(t=4.635, p<.001)$ and reported adverse event by others $(t=3.247, p=0.001)$ was a significantly positive correlation.

\section{Factors predicting patient safety competency}


A multiple stepwise regression analysis was performed using patient safety competency as the dependent variable and general characteristics, teamwork, and psychological safety as independent variables. Subscales of teamwork (team structure, leadership, communication, mutual support, situation monitoring), Psychological safety, Age, Gender, marital status, experience ED, Job Satisfaction, Education level, type of employment, Work Position, Training in patient safety, Experience in patient safety activity, reported adverse event by self and reported adverse event by others in sequence. Among these 18 variables, seven accounted for $40.9 \%$ of the variance in the final model $(F=24.291, p<$ .001). Thus, the significant predictors of patient safety competency were, Team structure $(\beta=0.374, p<$ $.001)$, Leadership $(\beta=0.188, p=0.012)$, Age $(\beta=0.177, p=0.003)$, type of employment $(\beta=-0.135, p=$ $0.022)$, Experience in patient safety activity $(\beta=0.128, p=0.014)$, Psychological safety $(\beta=0.125, p=$ $0.013)$ and gender $(\beta=0.105, p=0.037)$. Significant predictor variables of patient safety competency are shown in Table 4.

\section{Discussion}

In the emergency department, patient care depends on teamwork and coordination among clinical groups, because emergency nurses provide front-line care, patient safety competency is crucial to ensure quality safe care. In our opinion, this study is the first study that evaluates the level of teamwork and psychological safety and their relationship with patient safety competency among Iranian emergency nurses using valid tools and, therefore, adds new knowledge in this field.

The teamwork score among emergency nurses obtained in this study was slightly lower than that of Han \& Roh study (4) using similar tools. In another study in Iran, the score of teamwork among emergency nurses was slightly lower than our study (5). Nurses' perception of teamwork is related to patient safety (22). Teamwork leads to more employee job satisfaction, increased patient safety, improved quality of care, and greater patient satisfaction (9). Effective teamwork in healthy environments also helps care for high-quality patients (6). Besides, safe and quality patient care can also result from effective teamwork (9). Teamwork is the cornerstone of quality care and patient safety (7). The results of the study indicate the need to increase nursing teamwork at the study site. Emergency nurses can promote teamwork by facilitating communication, resolving disputes among team members, clarifying roles and responsibilities, and encouraging other team members.

The psychological safety of emergency nurses was lower than the results of a similar study in South Korea (4). Psychological safety plays a special role in high-risk work areas such as health care (13). When health care teams are psychologically safe, they are likely to improve the quality and teamwork initiatives (14). Psychological safety supports patient safety by contributing to quality improvement and encouraging employees to talk about mistakes (23). Promoting psychological safety among health care workers increases patient safety and improves the quality of health care. Therefore, promoting psychological safety among emergency nurses increases patient safety. Therefore, promoting the psychological safety of team members is important. Emphasis is placed on the role of leaders to create a 
safe psychological environment the psychological safety of team members is important. Emphasis is placed on the role of leaders in creating a safe psychological environment.

The level of patient safety competency in emergency nurses in this study was slightly higher than the findings of Han \& Roh (4) and lower than the findings of Langari et al. (17). These contradictory findings can be explained by cultural and field differences in patient safety education worldwide. The results of Han's study showed that emergency nurses focus mainly on patient-related emergency activities and have a relatively small understanding of patient safety. Therefore, they need patient safety management activities, training, standardization, and measures to cope with problems (4). Langari et al. (17) in their study showed that European countries (England and Finland) had started patient safety activities and training. They have also integrated patient safety into their nursing education programs. These measures have led to improved nurses' safety competency. Therefore, in Iran, patient safety qualifications are integrated into emergency nurses, and nursing trainers should provide a structured educational program to strengthen the patient's safety qualifications for vulnerable emergency nurses with low safety competency. Therefore, evaluating and improving the competency level of emergency nurses is essential to improve patient safety.

Among the organizational factors of team structure, leadership and psychological safety were among the main factors influencing the patient's safety competency. The emergency department is a potentially unique environment for teamwork and communication (10). The team structure is an integral part of the teamwork process. A properly structured patient care team is an enabler for and the result of effective communication, leadership, situation monitoring, and mutual support. Proper team structure can promote teamwork by including a clear leader, involving the patient, and ensuring that all team members commit to their roles in effective teamwork (6).

organized and effective teamwork in the emergency department has extensive implications on patient safety, quality of care, employee and patient satisfaction. A team with a proper teamwork structure can predict the requirements of other team members. It is dynamically compatible with a changing environment including changing team members' behavior and a common understanding of what should happen $(9,24)$. The nurses who consider their managers as strong leaders can empower their working environment, which, in turn, leads to their use of professional practice behaviors. These practical professional behaviors, such as effective communication, cooperation, and mutual understanding with clinical leadership's main characteristics, are compatible (25). The findings obtained by Boamah (24) showed that nurses often use clinical leader behaviors in their practice, which leads to the improvement of the patient's adverse events. To ensure the patient's safety, strong nursing leadership for implementation and continuity of effective management methods for training and continuous support for the environment is required to provide high-quality patient care. In our study, leadership has the largest teamwork score and is one of the predictors of patient safety competency. There are many factors, including leadership, communication, monitoring, and supporting behavior which helps create ideal teamwork in the emergency department (10). The findings of the study of Parr et al. show that leaders improve patients' satisfaction with patient services and safety (26). Based on the findings obtained by 
Kakemam et al. (22), nurses with stronger team leadership tend to report adverse events. Moreover, the result obtained by Kakemam showed that reducing medical errors can be achieved by effective leadership. Effective leadership can develop and strengthen the patient's safety and innovation culture in the health care environment. Accordingly, leaders are recommended to use educational strategies for reducing adverse events (22). The results obtained by Labrague et al. indicate the importance of the advancement of nurses' management in strengthening nurses' safety measures and reducing the results of the patient, and promoting the quality of nursing care (27). The results show that nursing leadership has a significant indirect effect on patient safety results. From a person-centered perspective, the care environment requires the empowerment of the workplace and effective relationships between leaders and nurses (28). Effective teamwork and leadership are recognized as important factors in many adverse events. Thus, a greater understanding of team dynamism and effectiveness and helping improve group training can lead to patient safety development and strengthen individual and team competencies in nontechnical aspects of care, such as prioritization, leadership, and decision making.

Having safe teams psychologically can improve learning, creativity, and performance in organizations. In the framework of health care, psychological safety supports patient safety by creating the ability to promote quality and encourage employees to talk about errors (23). Psychological safety has recently been identified as a necessity for effective teamwork $(23,29)$. Effective teamwork depends on the psychological safety of the team members, which are defined as their ability to trust each other and feel insufficient safety in the team to accept the fault, ask questions, present new data, or test new skills without fear of embarrassment or punishment. Higher psychological safety leads to better reports of adverse events (4). Strategies for increasing the psychological safety of emergency nurses in health care teams are required. When the employees are assured that the organization considers their welfare as the priority, he/she feels psychological safety and can talk about safety concerns, and this makes the patient's safety in everyday clinical performance (30). Providing psychological safety to emergency nurses increases patient safety and increases the quality of care. Therefore, nursing managers should always seek to develop effective methods to improve the psychological safety of their employees.

Old Age was the predictor of the patient safety competency of emergency nurses, such that the safety competency increases with increasing age. This finding was consistent with the results obtained by Alquwez and Chen $(31,32)$. This finding is probably due to the more professional and responsible behavior of the nurses with increasing age.

Experience in patient safety activities was another factor that affected the safety competency of emergency nurses. Nurses play a key role in coordinating patient safety activities because they are in close contact with patients and are involved in health care teams' decisions about patient safety (33). Having previous information and activities about patient safety can improve nurses' level of safety knowledge and affect their performance in patient safety (34). Therefore, nurses the more Experience in patient safety activities are in the emergency department, the more committed they will be to patient safety and compliance, and the more competent they will be in terms of patient safety. 
Gender was also one of the work-related factors predictors of the safety competency of emergency nurses. Hence, the mean score in women was significantly higher than men. These results were consistent with the results obtained by studies Chen and Jabarkhil $(32,35)$ and were not in line with the results presented in a study by Alquwez (31). This means that women pay attention to patient safety more than men do.

Emergency nurses with non-permanent employment status perceive patient safety competency more than those with permanent employment, which was not consistent with the study by Chegini et al. (36). It can be concluded that employed nurses are in the ultimate employment status. and contractual employees to upgrade to employed status have more efforts to improve their employment status. Therefore, they pay more attention to patient safety. it is necessary to provide in-service patient safety training for this group of employees.

\section{Limitation}

The current study had limitations to consider when using its results, including small sample size, probability of inaccuracy in answering questions due to time constraints in the emergency department and using a convenience sampling method. In the end, the interpretation of the reliability of the team's psychological safety should be under attention. Therefore, further studies with larger and random samples are recommended.

\section{Conclusion}

The work environment in emergency care is complex and unpredictable and may affect patient safety. This study's findings showed that the level of patient safety competency in emergency nurses is at a desirable level. Improving and upgrading team structure and leadership, having experience in patient safety activities, and improving and enhancing psychological safety at the nursing unit levels are essential to increase patient safety competencies in emergency nurses. It is recommended that patient safety training be provided to staff during service and that staff become familiar with patient safety activities and put patient safety at the forefront of all nursing activities and practices. Further studies are needed to evaluate the effects of an educational program designed to increase patient safety competencies in emergency nurses.

\section{References}

1. Han Y, Kim J-S, Seo Y. Cross-Sectional Study on Patient Safety Culture, Patient Safety Competency, and Adverse Events. Western Journal of Nursing Research. 2019;42(1):32-40.

2. Hosseini Marznaki Z, Pouy S, Salisu WJ, Emami Zeydi A. Medication errors among Iranian emergency nurses: A systematic review. Epidemiol Health. 2020;42:e2020030-e.

3. Kakemam E, Gharaee H, Rajabi MR, Nadernejad M, Khakdel Z, Raeissi P, et al. Nurses' perception of patient safety culture and its relationship with adverse events: a national questionnaire survey in 
Iran. BMC nursing. 2021;20(1):1-10.

4. Han JH, Roh YS. Teamwork, psychological safety, and patient safety competency among emergency nurses. Int Emerg Nurs. 2020;51:100892.

5. Ghezeljeh TN, Gharasoflo S, Haghani S. The relationship between missed nursing care and teamwork in emergency nurses: A predictive correlational study. Nursing Practice Today. 2020; 8(2):103-112

6. Rosen MA, DiazGranados D, Dietz AS, Benishek LE, Thompson D, Pronovost PJ, et al. Teamwork in healthcare: Key discoveries enabling safer, high-quality care. Am Psychol. 2018;73(4):433-50.

7. Rezaei S, Roshangar F, Rahmani A, Jabbarzadeh Tabrizi F, Sarbakhsh P, Parvan K. Emergency nurses attitudes toward interprofessional collaboration and teamwork and their affecting factors: A crosssectional study. Nursing and Midwifery Studies. 2021;10(3):173-180.

8. Hwang J-I. What are hospital nurses' strengths and weaknesses in patient safety competence? Findings from three Korean hospitals. International Journal for Quality in Health Care. 2015;27(3):232-238.

9. Costello M, Rusell K, Coventry T. Examining the average scores of nursing teamwork subscales in an acute private medical ward. BMC Nursing. 2021;20(1):84.

10. Grover E, Porter JE, Morphet J. An exploration of emergency nurses' perceptions, attitudes and experience of teamwork in the emergency department. Australas Emerg Nurs J. 2017;20(2):92-97.

11. Fay L, Carll-White A, Real K. Emergency nurses' perceptions of efficiency and design: Examining ED structure, process, and outcomes. Journal of emergency nursing. 2018;44(3):274-279.

12. O'Donovan R, McAuliffe E. Exploring psychological safety in healthcare teams to inform the development of interventions: combining observational, survey and interview data. BMC health services research. 2020;20(1):1-16.

13. Grailey KE, Murray E, Reader T, Brett SJ. The presence and potential impact of psychological safety in the healthcare setting: an evidence synthesis. BMC Health Services Research. 2021;21(1):773.

14. Edmondson AC. The fearless organization: Creating psychological safety in the workplace for learning, innovation, and growth: John Wiley \& Sons; 2018.

15. Tella S, Smith NJ, Partanen P, Jamookeeah D, Lamidi ML, Turunen H. Learning to ensure patient safety in clinical settings: comparing Finnish and British nursing students' perceptions. Journal of clinical nursing. 2015;24(19-20):2954-2964.

16. Ricklin ME, Hess F, Hautz WE. Patient safety culture in a university hospital emergency department in Switzerland-a survey study. GMS journal for medical education. 2019;36(2).

17. Langari MNM, Tella S, Smith N-J, Turunen H. Self-assessment of patient safety competence: A questionnaire survey of final year British and Finnish pre-registration nursing students. International Journal of Caring Sciences. 2017;10(3):1212.

18. Baker DP, Amodeo AM, Krokos KJ, Slonim A, Herrera H. Assessing teamwork attitudes in healthcare: development of the TeamSTEPPS teamwork attitudes questionnaire. Quality and Safety in Health Care. 2010;19(6):e49-e. 
19. Najafi M, Kohan N, Najafi M, Esmaily HM, Shirazi M. Assessment of Validity and Reliability of Attitudes to Health Professionals Questionnaire (AHPQ) in Iran. Research IN Medical Education. 2015;7(2):21-28.

20. Edmondson A. Psychological safety and learning behavior in work teams. Administrative science quarterly. 1999;44(2):350-383.

21. Shams G, KHALIJIAN S. The effect of secure-base leadership components on the staff psychological safety: the role of leadership effectiveness. 2014, 9(4), 33-53. [Persian].

22. Kakemam E, Hajizadeh A, Azarmi M, Zahedi H, Gholizadeh M, Roh YS. Nurses' perception of teamwork and its relationship with the occurrence and reporting of adverse events: A questionnaire survey in teaching hospitals. Journal of Nursing Management. 2021;29(5):1189-1198.

23. O’Donovan R, Van Dun D, McAuliffe E. Measuring psychological safety in healthcare teams: developing an observational measure to complement survey methods. BMC medical research methodology. 2020;20(1):1-17.

24. Boamah S. Linking Nurses' Clinical Leadership to Patient Care Quality: The Role of Transformational Leadership and Workplace Empowerment. Canadian Journal of Nursing Research. 2017;50(1):9-19.

25. PATRICK A, LASCHINGER HKS, WONG C, FINEGAN J. Developing and testing a new measure of staff nurse clinical leadership: the clinical leadership survey. Journal of Nursing Management. 2011;19(4):449-460.

26. Parr JM, Teo S, Koziol-McLain J. A quest for quality care: Exploration of a model of leadership relationships, work engagement, and patient outcomes. Journal of Advanced Nursing. $2021 ; 77(1): 207-220$.

27. Labrague LJ, Al Sabei SD, AbuAIRub RF, Burney IA, Al Rawajfah O. Authentic leadership, nurseassessed adverse patient events and quality of care: The mediating role of nurses' safety actions. Journal of Nursing Management. 2021;29(7):2152-2162.

28. Wang C, Horby PW, Hayden FG, Gao GF. A novel coronavirus outbreak of global health concern. The lancet. 2020;395(10223):470-473.

29. Parker H, du Plooy E. Team-based games: Catalysts for developing psychological safety, learning and performance. Journal of Business Research. 2021;125:45-51.

30. Rangachari P, L Woods J. Preserving organizational resilience, patient safety, and staff retention during COVID-19 requires a holistic consideration of the psychological safety of healthcare workers. International journal of environmental research and public health. 2020;17(12):4267.

31. Alquwez N, Cruz JP, Almoghairi AM, Al-otaibi RS, Almutairi KO, Alicante JG, et al. Nurses' perceptions of patient safety culture in three hospitals in Saudi Arabia. Journal of Nursing Scholarship. 2018;50(4):422-431.

32. Chen HY, Lu L, Ko YM, Chueh JW, Hsiao SY, Wang PC, et al. Post-Pandemic Patient Safety Culture: A Case from a Large Metropolitan Hospital Group in Taiwan. International Journal of Environmental Research and Public Health. 2021;18(9):4537. 
33. Oldland E, Botti M, Hutchinson AM, Redley B. A framework of nurses' responsibilities for quality healthcare - Exploration of content validity. Collegian. 2020;27(2):150-163.

34. Wake AD, Tuji TS, Gonfa BK, Waldekidan ET, Beshaw ED, Mohamed MA, et al. Knowledge, attitude, practice and associated factors towards patient safety among nurses working at Asella Referral and Teaching Hospital, Ethiopia: A cross-sectional study. PLOS ONE. 2021;16(7):e0254122.

35. Jabarkhil AQ, Tabatabaee SS, Jamali J, Moghri J. Assessment of Patient Safety Culture Among Doctors, Nurses, and Midwives in a Public Hospital in Afghanistan. Risk Manag Healthc Policy. 2021;14:1211-1217.

36. Chegini Z, Janati A, Afkhami M, Behjat M, Shariful Islam SM. A comparative study on patient safety culture among emergency nurses in the public and private hospitals of Tabriz, Iran. Nursing open. 2020;7(3):768-775.

\section{Tables}

Table 1. General characteristics of Emergency nurses $(\mathrm{N}=254)$. 


\begin{tabular}{|c|c|c|c|}
\hline Characteristics & Categories & Mean & SD \\
\hline Age (years) & & 31.70 & 6.30 \\
\hline \multirow[t]{2}{*}{ Job satisfaction } & & 3.35 & 1.27 \\
\hline & & $\mathrm{n}$ & $\%$ \\
\hline \multirow[t]{2}{*}{ gender } & male & 110 & 43.3 \\
\hline & female & 144 & 56.7 \\
\hline \multirow[t]{2}{*}{ Marital status } & Unmarried & 84 & 33.1 \\
\hline & Married & 170 & 66.9 \\
\hline \multirow[t]{2}{*}{ Education level } & Bachelor's degree & 211 & 83.1 \\
\hline & Master's degree and $\mathrm{PhD}$ & 43 & 16.9 \\
\hline \multirow[t]{3}{*}{ Type of employment } & Commitment & 56 & 22.0 \\
\hline & Contractual & 43 & 16.9 \\
\hline & Employed & 155 & 61.1 \\
\hline \multirow[t]{2}{*}{ Type of hospital } & General Hospital & 123 & 48.4 \\
\hline & specialized hospital & 131 & 51.6 \\
\hline \multirow[t]{3}{*}{ Type of emergency department } & Trauma Emergency & 88 & 34.6 \\
\hline & Internal emergency & 139 & 54.7 \\
\hline & Maternal and child emergency & 27 & 10.6 \\
\hline \multirow[t]{2}{*}{ Number of night shifts per month } & $\leq 6$ & 91 & 35.8 \\
\hline & $\geq 7$ & 163 & 64.2 \\
\hline \multirow[t]{3}{*}{ Total work experience (months) } & $\leq 60$ & 127 & 50.0 \\
\hline & $61-120$ & 73 & 28.7 \\
\hline & $\geq 121$ & 54 & 21.3 \\
\hline \multirow[t]{3}{*}{ Total work experience in emergency department (months) } & $\leq 60$ & 187 & 73.6 \\
\hline & $61-120$ & 49 & 19.3 \\
\hline & $\geq 121$ & 18 & 7.1 \\
\hline \multirow[t]{2}{*}{ Training in patient safety } & Yes & 187 & 73.6 \\
\hline & No & 67 & 26.4 \\
\hline \multirow[t]{2}{*}{ Experience in patient safety activity } & Yes & 154 & 60.6 \\
\hline & No & 100 & 39.4 \\
\hline \multirow[t]{2}{*}{ Reported adverse event by self } & Yes & 118 & 46.5 \\
\hline & No & 136 & 53.5 \\
\hline \multirow[t]{2}{*}{ Reported adverse event by others } & Yes & 157 & 61.8 \\
\hline & No & 97 & 38.2 \\
\hline
\end{tabular}

Table 2. Descriptive statistics and correlations among the study variables $(\mathrm{N}=254)$.

\begin{tabular}{|c|c|c|c|c|}
\hline \multicolumn{2}{|c|}{ Variable } & \multicolumn{3}{|c|}{ Patient safety competency } \\
\hline \multirow{6}{*}{ Teamwork } & Jeaddrchin & Mean \pm SD & Min/Max & $\begin{array}{c}r(p) \\
0.498(<0001)\end{array}$ \\
\hline & Communication & $3.51 \pm 0.62$ & $1-5$ & $0.409(<0.001)$ \\
\hline & Mutual support & $3.26 \pm 0.41$ & $1-5$ & $0.349(<0.001)$ \\
\hline & Situation monitoring & $3.75 \pm 0.75$ & $1-5$ & $0.447(<0.001)$ \\
\hline & Team structure & $3.65 \pm 0.77$ & $1-5$ & $0.571(<0.001)$ \\
\hline & total & $3.60 \pm 0.56$ & $1-5$ & $0.556(<0.001)$ \\
\hline \multicolumn{2}{|c|}{ Psychological safety } & $27.04 \pm 4.67$ & $9-39$ & $0.167(<0.001)$ \\
\hline \multirow{4}{*}{ Patient safety competency } & Knowledge & $2.94 \pm 0.65$ & $1-4$ & \\
\hline & Skills & $2.96 \pm 0.58$ & $1-4$ & \\
\hline & Attitudes & $3.00 \pm 0.53$ & $1-4$ & \\
\hline & total & $2.97 \pm 0.54$ & $1-4$ & 1 \\
\hline
\end{tabular}


Table 3. Relationship between nurses' general characteristics and patient safety competency. $(\mathrm{N}=254)$.

\begin{tabular}{|c|c|c|c|c|c|}
\hline Characteristics & Categories & Mean & SD & Test statistics & $\mathrm{p}$ value \\
\hline Age $\left(\right.$ years) ${ }^{a}$ & & 2.97 & 0.54 & 0.170 & 0.007 \\
\hline \multirow[t]{5}{*}{ Job satisfaction $^{\mathrm{c}}$} & very dissatisfied & 2.88 & 0.64 & 3.475 & 0.009 \\
\hline & A little dissatisfied & 2.91 & 0.37 & & \\
\hline & No idea & 2.77 & 0.63 & & \\
\hline & A little satisfied & 3.12 & 0.46 & & \\
\hline & very satisfied & 2.97 & 0.62 & & \\
\hline \multirow[t]{2}{*}{ Gender ${ }^{b}$} & male & 2.93 & 0.55 & -0.872 & 0.384 \\
\hline & female & 2.99 & 0.52 & & \\
\hline \multirow[t]{2}{*}{ Marital status ${ }^{b}$} & Unmarried & 2.95 & 0.57 & -0.354 & 0.723 \\
\hline & Married & 2.98 & 0.52 & & \\
\hline \multirow[t]{2}{*}{ Education level ${ }^{b}$} & Bachelor's degree & 3.02 & 0.51 & 3.290 & 0.001 \\
\hline & Master's and $\mathrm{PhD}$ & 2.72 & 0.61 & & \\
\hline \multirow[t]{3}{*}{ Type of employment ${ }^{c}$} & Commitment & 3.04 & 0.48 & 1.776 & 0.173 \\
\hline & Contractual & 3.06 & 0.49 & & \\
\hline & Employed & 2.92 & 0.57 & & \\
\hline \multirow[t]{2}{*}{ Type of hospital b } & generalized hospital & 2.79 & 0.50 & -5.224 & 0.000 \\
\hline & specialized hospital & 3.13 & 0.52 & & \\
\hline \multirow[t]{3}{*}{ Type of emergency department ${ }^{c}$} & Trauma Emergency & 3.09 & 0.52 & 3.602 & 0.029 \\
\hline & Internal emergency & 2.91 & 0.53 & & \\
\hline & Maternal and child & 2.86 & 0.62 & & \\
\hline \multirow[t]{2}{*}{ Number of night shifts per month ${ }^{b}$} & $\leq 6$ & 3.03 & 0.56 & 1.394 & 0.164 \\
\hline & $\geq 7$ & 2.93 & .52 & & \\
\hline \multirow[t]{3}{*}{ Total work experience (months) ${ }^{\mathrm{c}}$} & $\leq 60$ & 2.89 & 0.58 & 2.899 & 0.057 \\
\hline & $61-120$ & 3.01 & 0.47 & & \\
\hline & $\geq 121$ & 3.09 & 0.51 & & \\
\hline \multirow[t]{3}{*}{ Total work experience in emergency department (months) ${ }^{\mathrm{C}}$} & $\leq 60$ & 2.95 & 0.54 & 0.300 & 0.741 \\
\hline & $61-120$ & 3.01 & 0.48 & & \\
\hline & $\geq 121$ & 3.02 & 0.63 & & \\
\hline \multirow[t]{2}{*}{ Training in patient safety $b$} & Yes & 3.03 & 0.56 & 3.256 & 0.001 \\
\hline & No & 2.79 & 0.42 & & \\
\hline \multirow[t]{2}{*}{ Experience in patient safety activity ${ }^{b}$} & Yes & 3.09 & 0.56 & 4.635 & 0.000 \\
\hline & No & 2.78 & 0.45 & & \\
\hline \multirow[t]{2}{*}{ Reported adverse event by self ${ }^{b}$} & Yes & 3.04 & 0.55 & 1.917 & 0.056 \\
\hline & No & 2.91 & 0.52 & & \\
\hline \multirow[t]{2}{*}{ Reported adverse event by others ${ }^{b}$} & Yes & 3.05 & 0.54 & 3.247 & 0.001 \\
\hline & No & 2.83 & 0.50 & & \\
\hline
\end{tabular}

a Pearson r correlation. b t test for independent group. c Analysis of variance

Table 4. Stepwise multiple regression related to patient safety competency $(\mathrm{N}=254)$ 


\begin{tabular}{lcccccc}
\hline Variable & $B$ & $\mathrm{SE}$ & $\mathrm{Beta}$ & $\mathrm{t}$ & $p$ & $\Delta \mathrm{R}^{2}$ \\
\hline (Constant) & 0.831 & 0.296 & & 2.809 & 0.005 & \\
Team structure & 0.262 & 0.053 & 0.374 & 4.914 & $\mathrm{p}<0.001$ & 0.326 \\
\hline Leadership & 0.130 & 0.052 & 0.188 & 2.519 & 0.012 & 0.361 \\
\hline Experience in patient safety activity & 0.142 & 0.058 & 0.128 & 2.472 & 0.014 & 0.345 \\
\hline Psychological safety & 0.015 & 0.006 & 0.125 & 2.494 & 0.013 & 0.373 \\
\hline age & 0.015 & 0.005 & 0.177 & 2.966 & 0.003 & 0.386 \\
\hline Type of employment & -0.061 & 0.026 & -0.135 & -2.307 & 0.022 & 0.398 \\
\hline gender & 0.115 & 0.055 & 0.105 & 2.102 & 0.037 & 0.409 \\
\hline
\end{tabular}

AdjustedR $^{2}=0.39, \mathrm{~F}=24.291, p<0.001$ 